\title{
Updated Expressions for Determining Temperatures and Emission Measures from GOES Soft X-ray Measurements
}

\author{
Stephen M. White \\ Dept. of Astronomy, University of Maryland, College Park, MD 20742 USA \\ Roger J. Thomas and Richard A. Schwartz \\ Code 682, NASA/Goddard Space Flight Center, Greenbelt, MD 20771 USA
}

February 9, 2005

\begin{abstract}
We investigate the conversion of the $0.5-4$ and $1-8 \AA$ soft $\mathrm{X}$-ray flux measurements made by detectors on the Geostationary Operational Environmental Satellites (GOES) into temperature and emission measures of coronal plasma using modern spectral models and modern understanding of coronal abundances. In particular, the original analysis by Thomas, Starr \& Crannell (1985) is updated to take into account the realization that coronal abundances may be quite different from photospheric abundances. An important result of this analysis is that the derived temperatures and emission measures depend strongly on the assumed abundances even at high temperatures where continuum rather than spectral lines dominates the Sun's X-ray spectrum. This occurs because the higher coronal abundances mean that most of the continuum is due to free-bound emission processes, not free-free emission, and thus is abundance-dependent. We find significant differences between modern calculations of the temperature response of the flux measurements and the versions currently in use: for a typical flare, emission measures may be up to a factor of 4 smaller than the current software suggests. Derived temperatures are similar for both photospheric and coronal abundances for cool flares (e.g., $15 \mathrm{MK}$ ), but for hot flares (e.g., $35 \mathrm{MK})$ coronal abundances can lead to significantly $(\sim 25 \%)$ lower temperatures being derived.
\end{abstract}

Keywords: sample, $\mathrm{ATE}_{\mathrm{E}}$

\section{Introduction}

The National Oceanic and Atmospheric Administration (NOAA) launches and maintains a set of satellites called Geostationary Operational Environmental Satellites (GOES), carrying weather monitoring instruments. Each GOES satellite also carries a solar X-ray package (the "X-Ray Sensor", or XRS) consisting of a collimator that feeds a pair of ion chambers. These ion chambers measure the Sun's spatially integrated soft X-ray flux in two wavelength bands, 0.5-4 $\AA$ and 1-8 $\AA$, with a 3 -second cadence. The GOES soft X-ray detectors have provided an essentially uninterrupted monitor of the Sun's activity for 30 years, and are a valuable resource for the study of past solar activity and the prediction of space weather (e.g., Bornmann, 1990, Phillips and Feldman, 1995, Aschwanden and Alexander, 2001, Garcia, 2004).

For quantitative physical understanding of processes in the Sun's atmosphere, the X-ray fluxes themselves are of limited use. However, they reflect the temperature and emission measure of the plasma that produces the soft X-rays, and these physical quantities are of great importance: from them, the energetics of solar flares and other energy releases can be deduced, and their time variations can be interpreted directly in terms of physical models (e.g., Veronig et al., 2005). They can also be used for comparison with radio fluxes due to thermal bremsstrahlung. In order to use the GOES soft X-ray fluxes for such studies, Thomas et al. (1985) investigated the response of the two soft $\mathrm{X}$-ray bands as a function of the temperature of the radiating plasma and showed that the temperature and emission 
measure of hot plasma $(\sim 10 \mathrm{MK})$ can be derived in a straightforward fashion under the isothermal assumption: the ratio of the fluxes in the two GOES XRS bands unambiguously determines the (model-dependent) temperature, and once this is known the $\mathrm{X}$-ray flux in one of the bands determines the (model-dependent) emission measure.

Thomas et al. (1985) determined the temperature response by folding model X-ray spectra of isothermal plasmas through the response of the GOES 1 XRS detectors and produced handy polynomial approximations that have been widely used to derive temperatures and emission measures from GOES fluxes. These quantities are volume-averaged, since they have no spatial resolution, but, e.g., during flares the flare emission can easily be isolated by background subtraction and the quantities so derived are physically representative. Since they are obtained from just two numbers, they cannot accomodate the widely recognized multi-thermal nature of the solar corona, i.e., the fact that at any given time the corona is inhomogeneous and different temperature plasmas are present at different locations. However, for topics such as overall energetics, the volume-averaged quantities represented by the GOES XRS data remain important tools.

The motivation to revisit the determination of temperature and emission measure from GOES XRS data comes from two notable developments. The first is improvements in modelling of solar X-ray spectra that have taken place. Spectra in the relevant temperature range (roughly 5-30 MK) contain both continuum and spectral lines, and depend on the ionization equilibrium of each atomic species as well as the transition strengths of the lines. Both these topics are much better understood than was the case in 1985. Secondly, and probably more importantly, is the recognition that the abundance distribution in the solar corona need not be the same as in the solar photosphere. At about the time of the Thomas et al. (1985) analysis it was starting to be recognized that abundance distributions actually depend on the amount of energy required to remove the outermost electron from an element's neutral atom. This so-called "FIP-effect" (for first ionization potential) results in low-FIP elements, such as Fe, having a higher abundance in the corona relative to high-FIP elements, such as O, than they do in the photosphere (Meyer, 1985). Because of the difficulty of measuring absolute abundances, debate as to whether low-FIP elements were enhanced in the corona or high-FIP elements were depleted in the corona (relative to the photosphere) continued for some time. However, a concensus seems to have emerged that high-FIP elements have the same absolute abundance in the corona as in the photosphere, while the low-FIP elements are enhanced in the corona by a factor of order 4 (Feldman, 1992; Feldman and Laming, 2000; White et al., 2000). The picture is complicated by evidence that the enhancement factor is not steady, but varies with time and location and may depend, for example, on the age of an active region (Fludra and Schmelz, 1999; Schmelz, 1999; Feldman and Widing, 2003).

There have been other analyses of the GOES XRS data since Thomas et al. (1985). Garcia (1994) describes the operation of the GOES XRS detectors in great detail, and particularly the differences between satellites. He used spectra calculated with both the Raymond (Raymond and Smith, 1977) and Mewe (Mewe et al., 1985) models to determine the temperature response. The nature of the abundances and ionization equilibrium used is not described. Sylwester et al. (1995) extend Garcia's analysis, but also did not investigate the role of abundances. R. A. Schwartz implemented the differences between detectors on different satellites discussed in these papers in the goes routine in the SolarSoft package (Freeland and Handy, 1998), and used model spectra from the SolarSoft task mewe_spec, which is based on the Mewe model with Meyer coronal abundances (Meyer, 1985), to determine the temperature response of the GOES XRS detectors. 


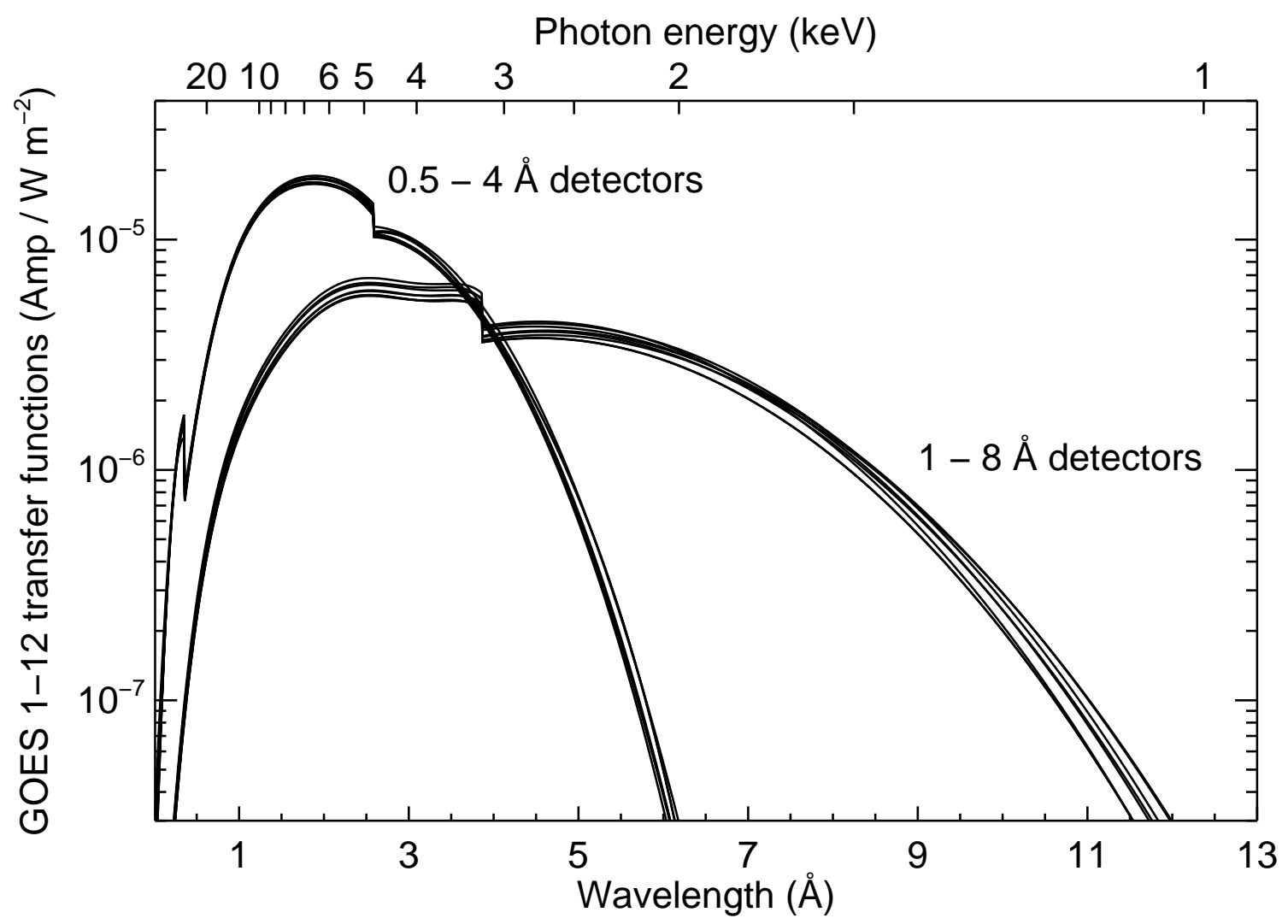

Figure 1. The "transfer functions" $\mathrm{G}(\lambda)$ for the $0.5-4$ and $1-8 \AA$ XRS detectors on GOES satellites 1 to 12 , overplotted without distinction. This plot indicates the variation in the response of the detectors on the different satellites.

In this paper we repeat the analysis of Thomas et al. (1985), Garcia (1994) and Sylwester et al. (1995) using modern model spectra from the CHIANTI database (Dere et al., 1997; Young et al., 1998), and compare the results for both coronal and photospheric abundances with the earlier work, and with the GOES analysis software that has been available in the SolarSoft package. We find significant differences, primarily resulting from the abundance issue. However, it may be surprising that the main effect is not in the spectral lines, but rather in the free-bound contribution to the continuum emission. This can be larger than the free-free emission in the relevant temperature range, and therefore the effect is still important at higher temperatures where the spectral lines are less significant.

\section{GOES XRS Transfer Functions}

The detailed nature of the response of the GOES XRS detectors to soft X-rays is described by Thomas et al. (1985) and Garcia (1994). The raw measured quantities are currents induced in the ion chambers by the incident X-rays. The detectors are calibrated prior to launch. The "transfer functions" that represent the measured response (detector current per incident $\mathrm{X}$-ray flux) as a function of wavelength are shown in Figure 1 for GOES 1-12 (these are the functions used in the GOES software routines in SolarSoft). The point of this figure is 


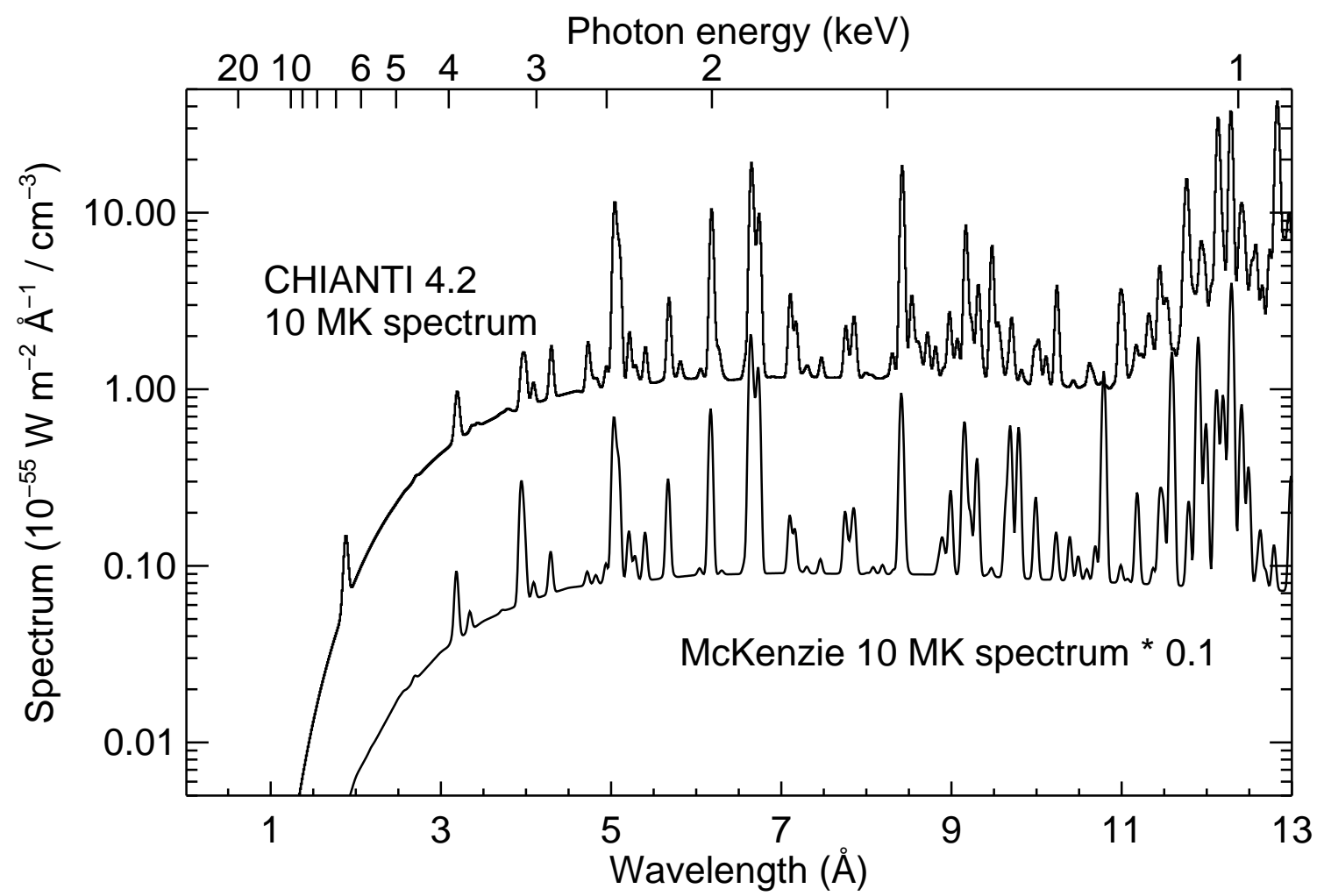

Figure 2. A comparison of the model spectrum for a $10 \mathrm{MK}$ plasma obtained from CHIANTI 4.2 with photospheric abundances (upper curve) with the $10 \mathrm{MK}$ spectrum calculated by McKenzie (lower curve) and used by Thomas, Starr \& Crannell (1985) in their original calculations of the GOES XRS temperature response. The McKenzie spectrum has been divided by 10 to offset it from the CHIANTI spectrum. The spectral resolution is $0.05 \AA$. The wavelength scale is shown on the bottom axis and the corresponding photon energy scale on the top axis.

that there are differences between detectors on the different satellites, but they are relatively small.

The actual X-ray fluxes incident on the detectors are not known since different spectral forms can produce the same currents. The reported $\mathrm{X}$-ray fluxes are derived by assuming that a wavelength-averaged response is appropriate for each detector. The coefficients used to convert the currents measured in the ion chambers into the reported $\mathrm{X}$-ray fluxes can differ significantly from one satellite to the next. These coefficients, labelled $\overline{\mathrm{G}}_{4}(0.5-4 \AA$ channel) and $\overline{\mathrm{G}}_{8}$ (1-8 $\AA$ channel), are tabulated in the appendix (we thank Rodney Viereck of NOAA for supplying the previously unavailable calibration data for the XRS detectors on the GOES 12 satellite). They represent wavelength-averaged transfer functions (see Garcia 1994 and Thomas et al. 1985), in units of Amp/( $\left.\mathrm{W} \mathrm{m}^{-2}\right)$.

$\overline{\mathrm{G}}_{4}$ varies by up to $40 \%$ from early satellites to later versions, mostly due to a decision to normalize the shorter-wavelength channel over the range $0.5-3 \AA$ rather than $0.5-4 \AA$ (discussed by Garcia 1994), while $\overline{\mathrm{G}}_{8}$ can differ by $20 \%$. The different values of the $\overline{\mathrm{G}}$ coefficients affect the temperature response in terms of the $\mathrm{X}$-ray fluxes. This is discussed in the next section. However, we emphasize that it is only the derived temperatures and emission measures that depend on the models used to represent X-ray spectra from hot plasma, not the actual currents in the ion chambers themselves. 


\section{Spectral Models}

To determine the temperature response of the reported GOES XRS fluxes, we convolve the wavelength-dependent transfer functions in Figure 1 with models of the X-ray spectrum of an isothermal plasma and convert them to GOES X-ray fluxes using the formalism described by Thomas et al. (1985) and Garcia (1994) with $\overline{\mathrm{G}}$ coefficients from Table I. We use models calculated using version 4.2 of the CHIANTI package ${ }^{1}$ (Landi et al., 1999; Landi et al., 2002). Ionization equilibrium is determined using the Mazzotta et al. (1998) calculations as implemented in CHIANTI. Continuum from free-free, free-bound and two-photon processes is included (Landi et al., 1999). Spectra from 0.01 to $20 \AA$ were calculated with $0.01 \AA$ wavelength bins for 41 temperatures logarithmically spaced from 1.0 to $100.0 \mathrm{MK}$ using both the solar coronal abundances and the solar photospheric abundances incorporated in CHIANTI. A density of $10^{10} \mathrm{~cm}^{-3}$ was assumed for the calculations; we compared spectra at $10 \mathrm{MK}$ for densities of $10^{9} \mathrm{~cm}^{-3}, 10^{10} \mathrm{~cm}^{-3}$ and $10^{11} \mathrm{~cm}^{-3}$, and found no significant differences between them, so our results should be essentially independent of the assumed density, at least over that range.

To illustrate developments in spectral modelling since the Thomas et al. (1985) work, in Figure 2 we compare the CHIANTI 4.2 spectrum at $10 \mathrm{MK}$ with the spectrum used by Thomas et al. (1985) at 10 MK. For their temperature-dependence calculations, Thomas et al. (1985) used spectra provided by McKenzie based on calculations by Walker (see Walker, Jr. et al., 1974) with other supporting material, and used ionization-balance calculations by Jordan (1970). These spectra do not show all the lines found in modern CHIANTI spectra, but most of the strong lines are present and the continuum has the same shape. An obvious difference between the older McKenzie spectrum and the modern spectrum is in the forest of Fe L-shell lines that form almost a bright quasi-continuum from $12-15 \AA$ in the CHIANTI spectrum (Liedahl et al., 1995; Savin et al., 1996; Gu et al., 2001; Brown et al., 2002), where the McKenzie spectrum is much weaker. However, these lines are outside the main wavelength sensitivity of the GOES XRS 1-8 $\AA$ channel (Fig. 1) and so this difference does not affect significantly the quantities derived from the GOES XRS fluxes.

To compare the continuum and the effects of the assumed abundances in more detail, Figure 3 shows the coronal and photospheric abundance CHIANTI 10 MK spectra and the McKenzie $10 \mathrm{MK}$ spectrum in the 3-6 $\AA$ range. The continuum in the coronal abundance CHIANTI spectrum is considerably higher than in the photospheric abundance CHIANTI spectrum, which in turn is about $10 \%$ higher than in the McKenzie spectrum. Inspection of the lines that are common to all three spectra indicates that the McKenzie spectra were probably calculated with abundances close to solar photospheric values, which is what we would expect for that epoch. We also compared these spectra with the results of the mewe spec code in SolarSoft, which uses the Mewe models and assumes "Meyer coronal" abundances (Meyer, 1985) in which the low-FIP lines are at solar photospheric values and high-FIP lines are depressed relative to their photospheric abundances. This spectrum has a continuum level very similar to the photospheric abundance CHIANTI spectrum (as it should since the CHIANTI continuum calculation is based on the Mewe calculation) and, as expected, highFIP lines (demonstrated by the labelled $\mathrm{S}$ lines) that are weaker than their counterparts

\footnotetext{
1 CHIANTI 5.0 is due to be released in early 2005 and includes some additional lines in the 8-11 $\AA$ region not present in 4.2. We have compared a CHIANTI 5.0 spectrum for a 10 MK plasma supplied by Enrico Landi with the CHIANTI 4.2 spectrum used here and find that it produces a GOES XRS response that differs by of order $5 \%$ from our results. The CHIANTI 5.0 models will be used in the SolarSoft analysis software once they become available.
} 


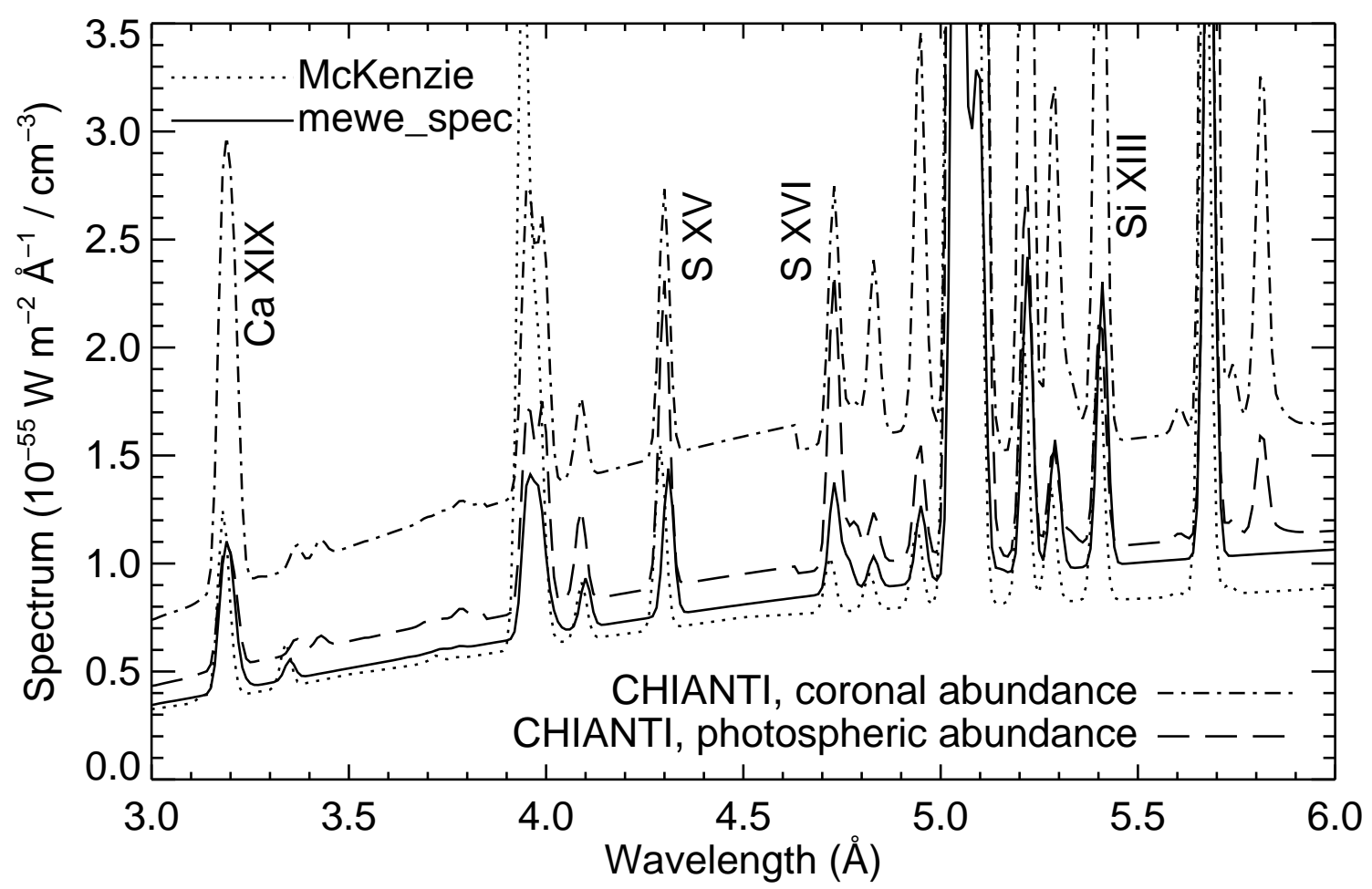

Figure 3. Comparison in the 3-6 A range of model spectra at 10 MK: the CHIANTI 4.2 spectrum with coronal abundances (dot-dashed line), the CHIANTI 4.2 spectrum with photospheric abundances (long-dashed line), the mewe_spec spectrum presently used in the SolarSoft goes task (solid line), and the McKenzie spectrum used by Thomas, Starr \& Crannell (1985) (dotted line). The spectral resolution is $0.03 \AA$ in all spectra. Four individual lines are labelled: low-FIP Ca and Si lines, and high-FIP S lines. In this figure the vertical scale is linear and all spectra are plotted on the same scale.

in the CHIANTI photospheric-abundance and coronal-abundance spectra. By contrast, the low-FIP Ca and Si lines have similar strength in the CHIANTI photospheric-abundance and mewe_spec spectra, but are much stronger in the CHIANTI coronal-abundance spectrum.

The reason for the much higher continuum level in the coronal-abundance spectrum is the dominance of free-bound emission in this wavelength range. Figure 4 shows the relative contributions of free-bound, free-free and 2-photon continua for coronal and photospheric abundances at $10 \mathrm{MK}$ (see also Figure 6 of Young et al., 2003, which is the same plot but for the photospheric abundance case only). The free-bound emission depends on the higher-Z elements and therefore is abundance-dependent: for coronal abundances, the freebound emission at this temperature is about twice the free-free emission below $6 \AA$. Just as importantly, because the free-bound continuum peaks at shorter wavelengths, it pushes the peak of the total continuum emission to shorter wavelengths at temperatures above 10 MK. This increases the flux in the $0.5-4 \AA$ channel relative to the $1-8 \AA$ channel for the same temperature, and thus in some temperature ranges (above $15 \mathrm{MK}$ ) the ratio of the flux in the $0.5-4 \AA$ channel $\left(B_{4}\right)$ to the flux in the 1-8 $\AA$ channel $\left(B_{8}\right)$ will be larger for a given temperature if the emitting plasma has coronal rather than photospheric abundances (see also Fig. 7). Note that if one replots Figure 4 for other temperatures one finds that as temperature increases the free-free continuum emission increases faster than the free-bound, 


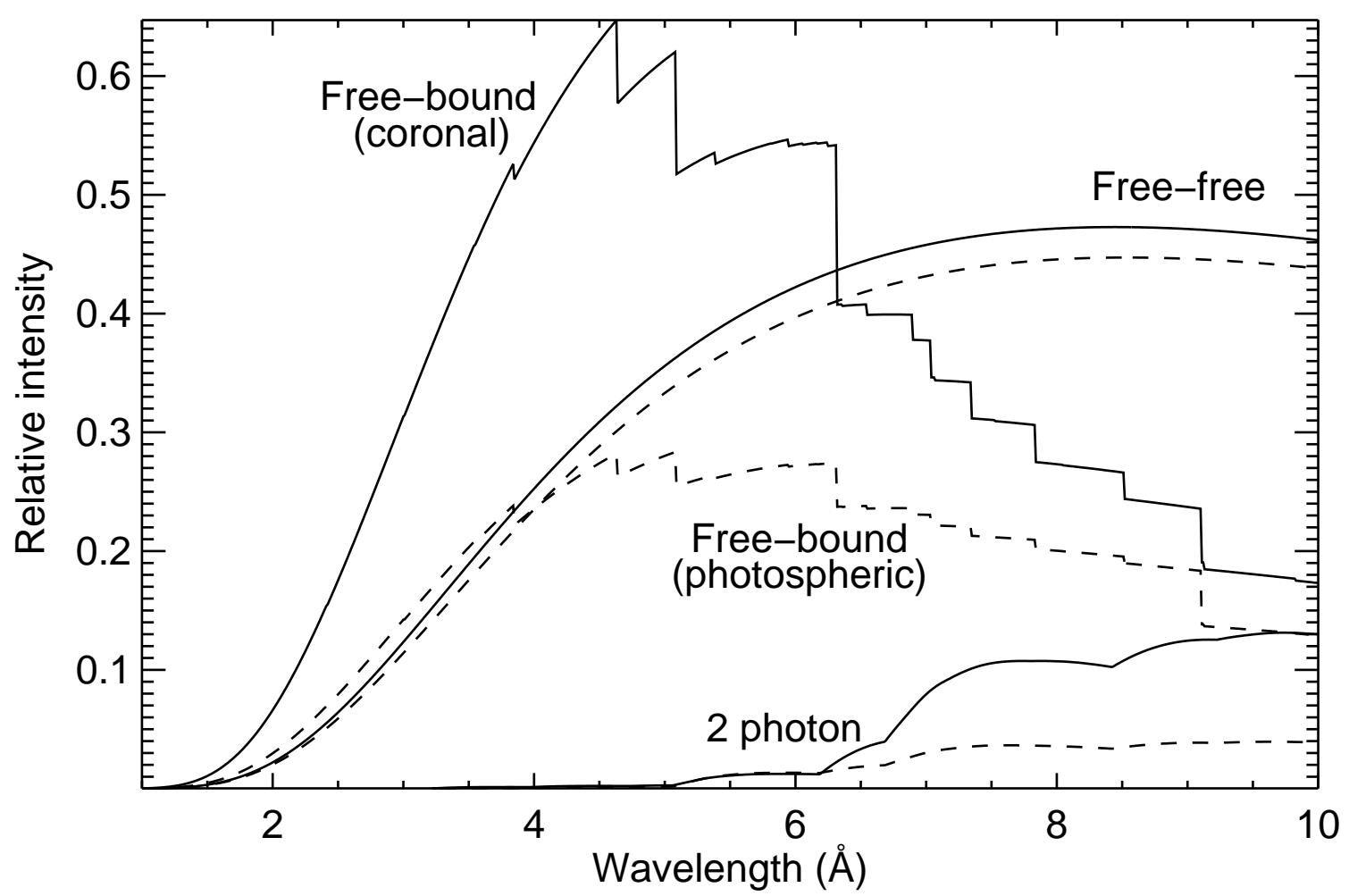

Figure 4. Comparison of the three contributions to the continuum level in CHIANTI 4.2 isothermal spectra at $10 \mathrm{MK}$. The free-bound, free-free and 2-photon continua for coronal abundances (solid lines) and photospheric abundances (dashed lines) are labelled. The curves are normalized to the peak intensity of the total coronal-abundance continuum emission (the sum of the three solid-line curves plotted). The sharp edges in the free-bound continuum spectra represent transitions to different charge states of common ions.

so that for coronal abundances the two contributions to the continuum are roughly equal at $20 \mathrm{MK}$.

It is sometimes assumed that at high temperatures the abundance issue is irrelevant for the conversion of GOES XRS fluxes to temperature and emission measure because the continuum rather than the spectral lines dominates the spectrum: this impression is incorrect for coronal abundances because free-bound emission (which is abundance-dependent) rather than free-free emission (which is not) dominates the continuum. Figure 5 presents the relative contribution of spectral lines to the total flux in the two GOES XRS channels for the assumptions of coronal (solid lines) and photospheric (dashed lines) abundances (cf. Fig. 5 of Thomas et al., 1985). For photospheric abundances the lines produce less than $20 \%$ of the X-ray flux above $20 \mathrm{MK}$ in either GOES channel, but for coronal abundances the $0.5-4$ $\AA$ channel can have over $30 \%$ of its flux above $20 \mathrm{MK}$ due to lines. This is almost entirely contributed by the Fe XXV/XXIV complex at $1.85 \AA$, close to the peak in the response of the $0.5-4 \AA$ channel. We note that Phillips (2004) has recently discussed the use of this line complex in conjunction with adjacent continuum to investigate the Fe abundance of flares.

It is also useful to consider the energy ranges of the photons that contribute to the GOES soft X-ray fluxes. The nominal wavelength ranges of $0.5-4 \AA$ and $1-8 \AA$ correspond roughly to 3-25 keV and 1.5-12 keV, but, e.g. a temperature of $10 \mathrm{MK}$ corresponds to a photon energy of order $1 \mathrm{keV}$, and such a spectrum has essentially no photons above a few keV (e.g., see Fig. 2), so the upper energy limits quoted above are usually misleading. In Figure 6 we plot the mean 


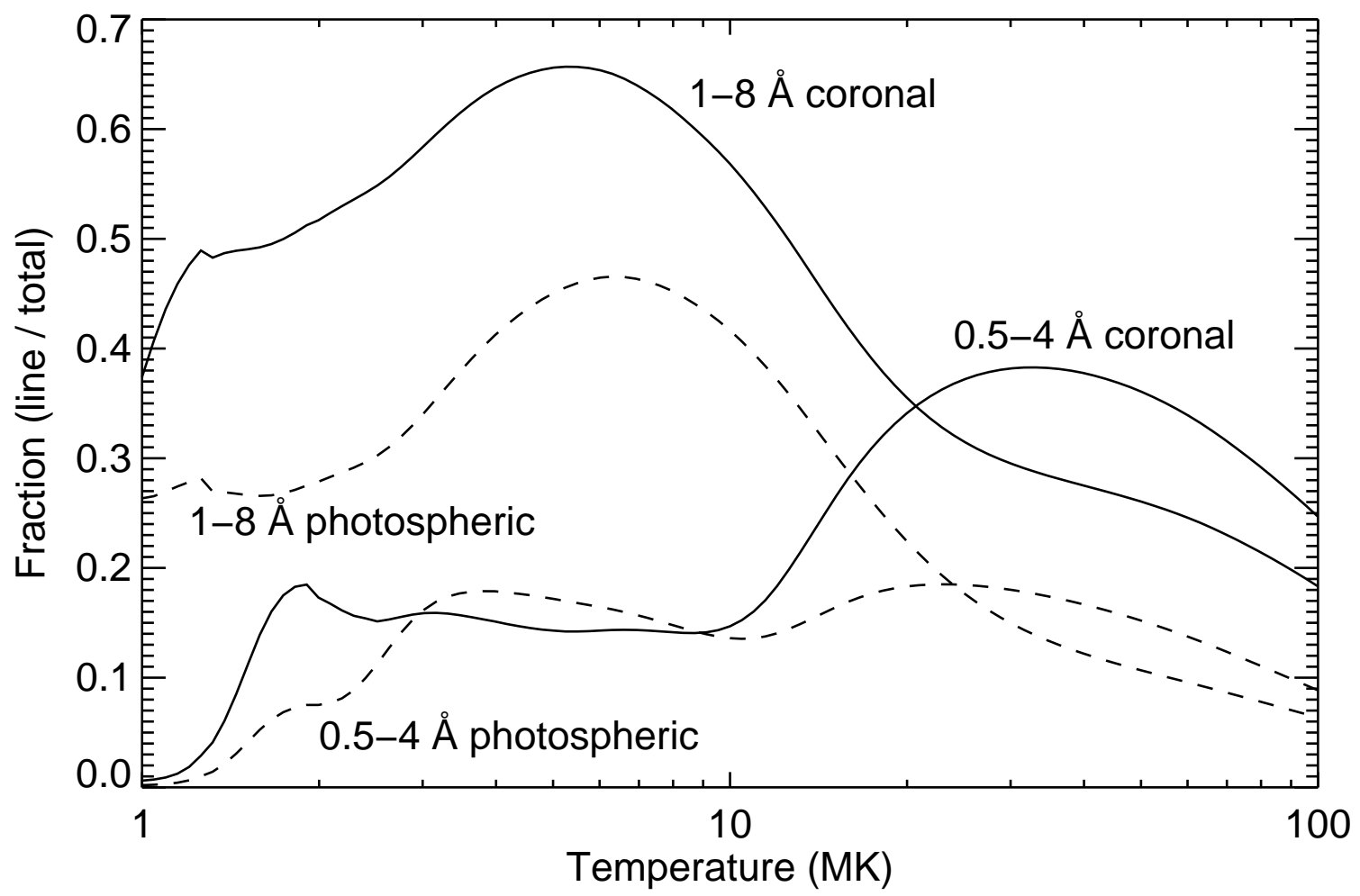

Figure 5. The relative contribution of spectral lines to the reported X-ray fluxes in the two GOES XRS channels as a function of temperature for coronal abundances (solid curves) and photospheric abundances (dotted curves). This calculation was carried out for the GOES 10 responses using spectra from CHIANTI 4.2 .

photon energy (i.e., averaged using the product of the appropriate transfer function with a model spectrum) and the $\pm 1 \sigma$ energy limits (i.e., the energy range that produces the central $68 \%$ of the total contribution to the flux) for each of the GOES XRS channnels as a function of temperature, derived using the CHIANTI 4.2 coronal and photospheric abundance models. Below $20 \mathrm{MK}$ the two GOES XRS channels are dominated by quite different typical photon energies (the contributing energy ranges are separated), with the shorter-wavelength channel seeing more energetic photons as expected, but above $20 \mathrm{MK}$ the model spectra tend to be dominated by emission in the 1-2 $\AA(6-12 \mathrm{keV})$ range that contributes to both XRS channels and this reduces the ratio of the mean photon energies contributing to the two channels. The energy range of the photons contributing most to the GOES XRS response is typically about $4 \mathrm{keV}$ in both channels at higher temperatures. The results are very similar for both coronal and photospheric abundances, but there is a small difference in the width of the energy range contributing to the 0.5-4 $\AA$ channel at higher temperatures, where the narrow Fe emission feature at $1.85 \AA$ is four times brighter in the coronal abundance spectrum than in the photospheric abundance spectrum and is responsible for a smaller effective width.

\section{GOES XRS Temperature and Emission Measure Response}

We follow the scheme introduced by Thomas et al. (1985) for deriving the temperature, $T$, and volume emission measure, $E M=N_{e} N_{H} V$ where $N_{e}$ is the electron density, $N_{H}$ is the 

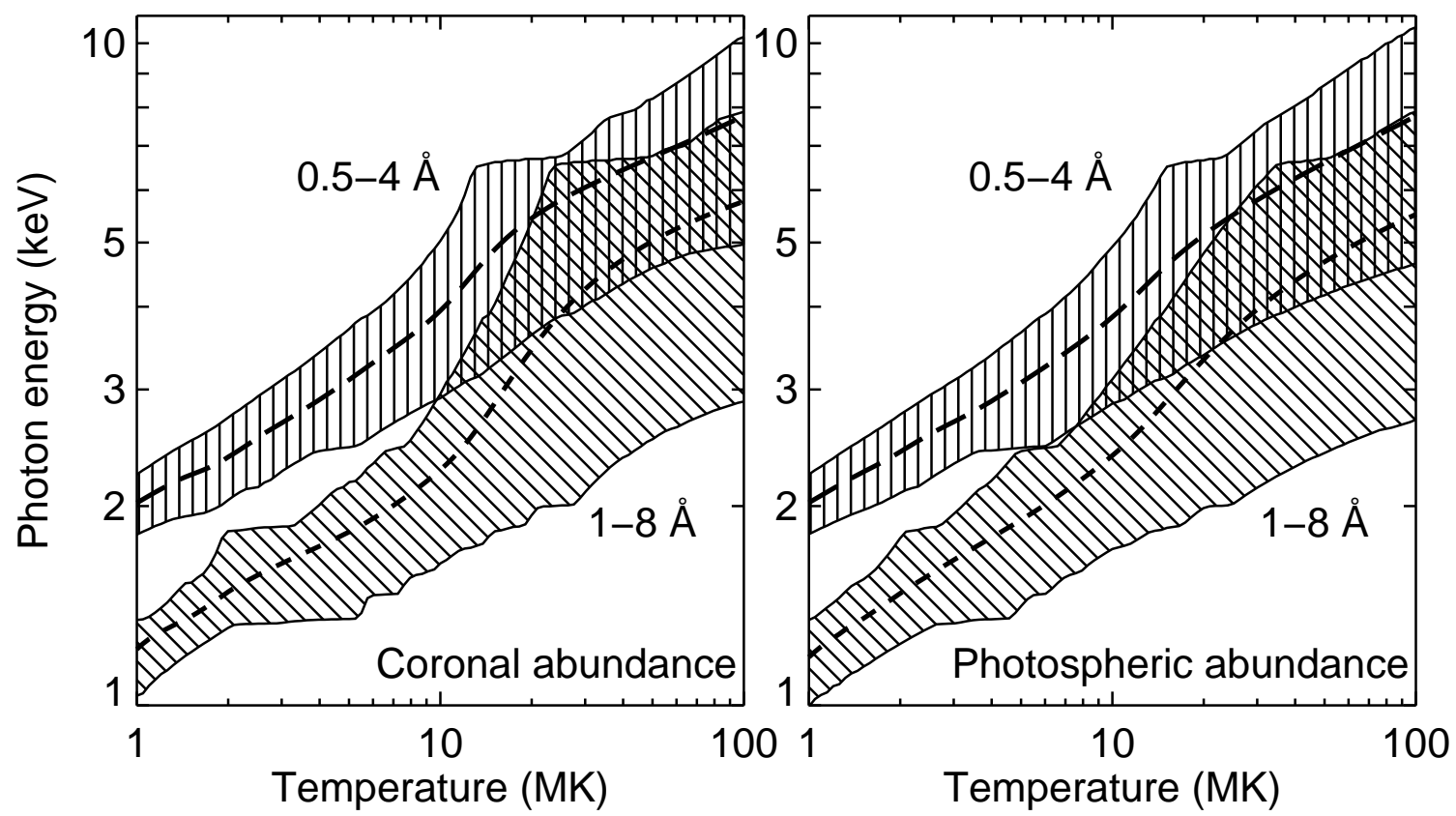

Figure 6. Plots of the photon energy ranges contributing to the the GOES X-ray responses in each channel as a function of temperature for coronal abundances (left panel) and photospheric abundances (right panel). The shaded regions indicate the $\pm 1 \sigma$ limits of the photon energies contributing to the $0.5-4 \AA$ channel (vertically shaded region) and the 1-8 $\AA$ channel (obliquely shaded region). The dashed lines show the mean photon energy contributing to each channel: the mean energy is the photon energy weighted by the product of the spectrum and the appropriate transfer function. The limits of the contributing energy range are defined to be the $16 \%$ and $84 \%$ levels in the cumulative contribution function. The plot shows results for the GOES 10 detectors.

proton density and $V$ is the volume of the (assumed homogeneous and isothermal) source, from the reported $\mathrm{X}$-ray fluxes $B_{4}(0.5-4 \AA$ channel $)$ and $B_{8}(1-8 \AA$ channel). Since the ratio of the levels in the two channels $R=B_{4} / B_{8}$ is a monotonic function of temperature over the range 1-100 MK, it can be inverted to determine the temperature. Once the temperature is known, the emission measure is derived from the reported flux in the 1-8 $\AA$ channel using a temperature-dependent scaling factor. The scaling between $R$ and $T$, and between $B_{8}(T)$ and $E M$, is determined by calculating GOES XRS responses in the two channels for the model data described in the preceding section. The same approach is employed in the SolarSoft goes widget.

The detailed response in terms of fluxes in the two channels varies from one satellite to the next, as described above. In Figure 7 we show as an example the response for the GOES 10 XRS detectors. The upper panel of the figure shows the dependence of temperature on the flux ratio for five different responses: the CHIANTI spectrum with coronal abundances (solid line), the CHIANTI spectrum with photospheric abundances (long dashes), the Mewe spectrum with Meyer (1985) abundances used in goes in SolarSoft (dash-dots), the original McKenzie spectra used by Thomas et al. (1985) (short dashes), and the Thomas et al. (1985) polynomial approximation (dotted line). The temperature response is very similar for the three curves in which low-FIP abundances are at photospheric levels (CHIANTI/photospheric, Mewe, McKenzie). As noted in the last section, at higher temperatures the coronal abundance curve produces cooler temperatures for a given $B_{4} / B_{8}$ flux ratio due to the shift in the location of the peak wavelength of the continuum emission. 

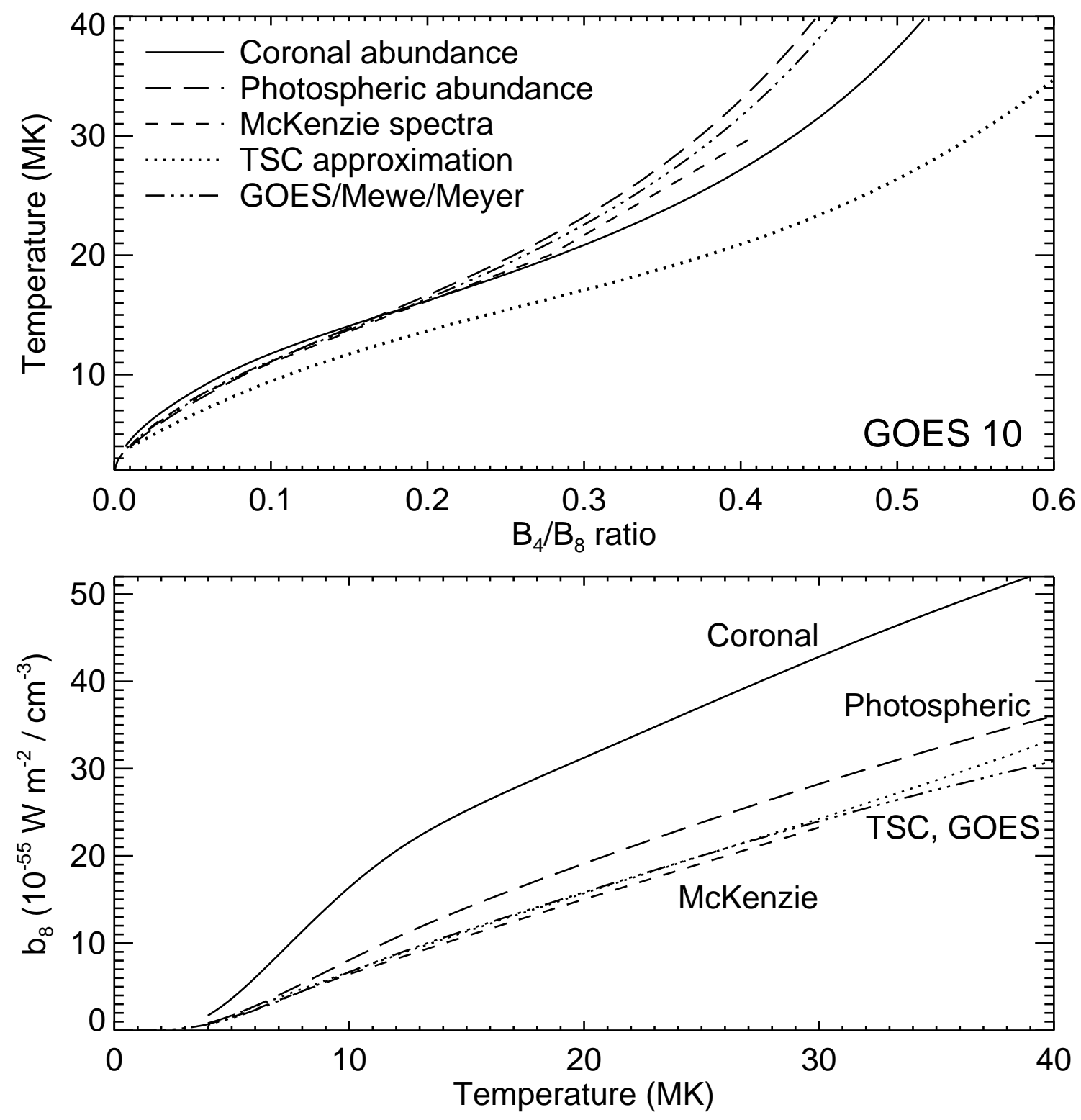

Figure 7. Plots of the temperature and emission measure response of the XRS detectors on the GOES 10 satellite for five different sets of models. The short dashed line is derived from the McKenzie spectra, while the dotted line represents the polynomial approximation derived by Thomas, Starr \& Crannell (1985) for the GOES 1 satellite. The dash-dot line labelled "GOES/Mewe/Meyer" is the response for the Mewe spectrum with Meyer (1985) abundances: this is the response used currently in the goes widget in SolarSoft. The long dashed line is the response for CHIANTI 4.2 spectra with photospheric abundances, while the solid line is for CHIANTI 4.2 spectra with coronal abundances. $B_{4} / B_{8}$ is the ratio of the reported $\mathrm{X}$-ray fluxes in the two channels, and $b_{8}$ is the normalized response of the 1-8 $\AA$ channel (flux per unit emission measure). Labels in the lower panel are abbreviated versions of the labels in the upper panel.

The Thomas et al. (1985) polynomial approximation does not resemble any of the other curves for GOES 10. This will be the case when applying the the original Thomas et al. (1985) polynomial approximation to data from any satellite after GOES 3, since the Thomas et al. (1985) formalism was based on a definition of the $\overline{\mathrm{G}}_{4}$ coefficient that was altered starting 


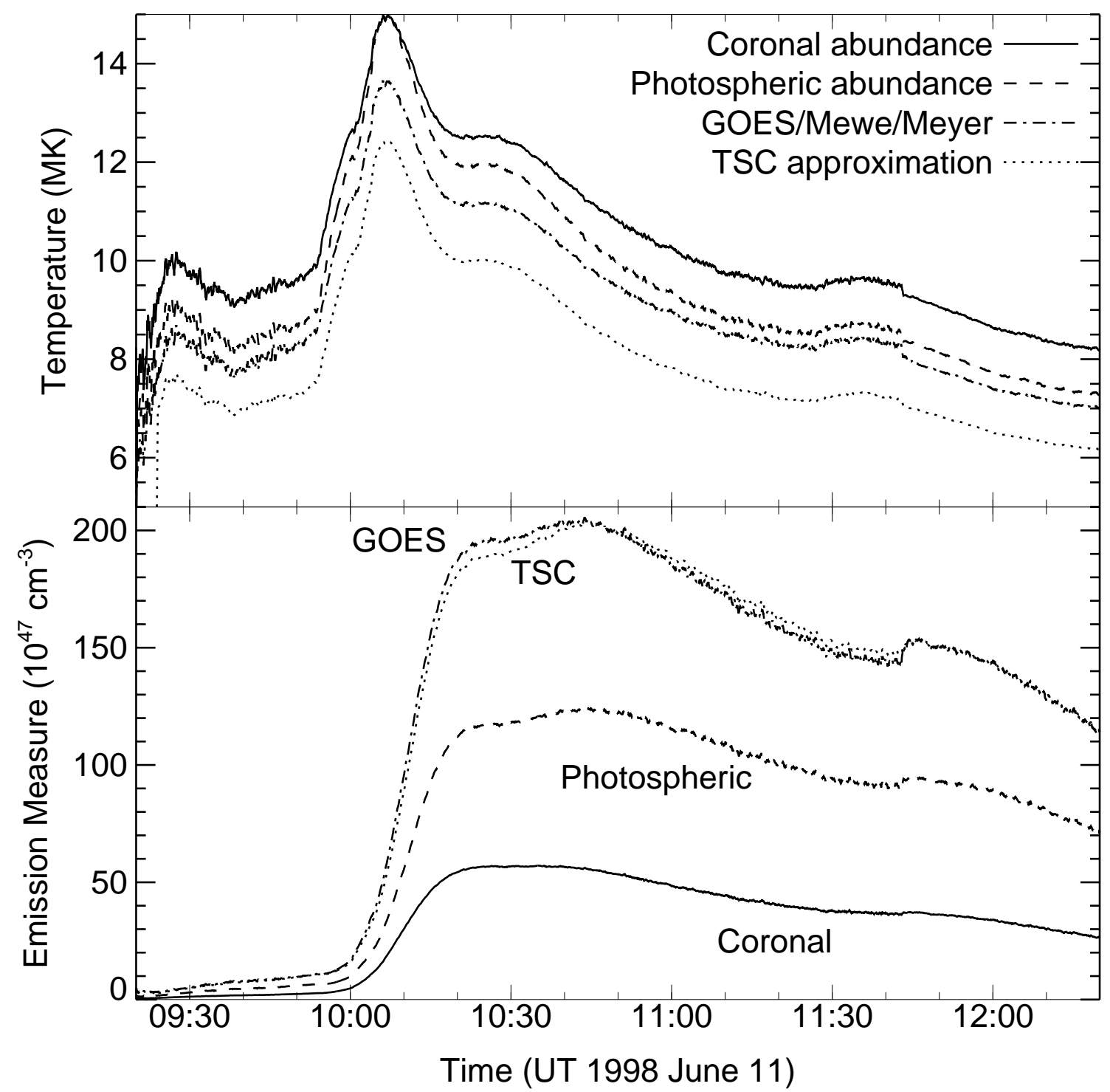

Figure 8. Comparison of the temperature (upper panel) and emission measure (lower panel) derived from GOES soft X-ray data for a flare on 1998 June 11. The four curves are the CHIANTI model with coronal abundances (solid line), the CHIANTI model with photospheric abundances (dashed line), the Mewe model with Meyer abundances as used in the goes widget in SolarSoft (dot-dashed line), and the Thomas et al. polynomial approximation (dotted line). The data (and the responses used) are from the GOES 8 detectors.

with GOES 4 (as can be seen in Table I). The effect of that rescaling is to cause a given detected ion-chamber current in the short-wavelength channel of GOES 4-12 to be reported as a smaller soft X-ray flux than if it had been measured by GOES 1-3. A better agreement with the Thomas et al. (1985) approximation is obtained if the reported 0.5-4 $\AA$ fluxes are multiplied by an empirical factor of 1.631/1.16 (Table I) before application of the formalism. For GOES 1-3 we find that the Thomas et al. (1985) polynomial approximation is indeed close to the responses to photospheric-abundance CHIANTI models at temperatures below $15 \mathrm{MK}$. 
The lower panel of Figure 7 shows the relationship between the 1-8 $\AA$ response per unit emission measure, $b_{8}=\left(10^{55} \mathrm{~cm}^{-3} / E M\right) B_{8}$, and the temperature. The Mewe, McKenzie and Thomas et al. (1985) polynomial approximation all have a very similar response, being quite close to linear in temperature as in Figure 4 of Thomas et al. (1985). The CHIANTI/photospheric curve shows a slightly larger response, while the CHIANTI/coronal response is substantially larger and has a noticeably different shape, with a steeper slope below $10 \mathrm{MK}$ than above. The impact of the larger response to the CHIANTI coronal spectrum is that a smaller (hydrogen) emission measure is needed to produce a given reported GOES XRS flux value compared with the other spectral models considered.

For completeness and to demonstrate the quantitative nature of the differences in response, we have determined polynomial approximations to the response curves for the CHIANTI model spectra demonstrated in Fig. 7. Coefficients for each satellite up to GOES 12, for the cases of both coronal and photospheric abundances, are given in Tables II and III in the appendix, valid in the temperature range from 5 to $30 \mathrm{MK}$ that encompasses most flare emissions detected by GOES. These tables, particularly Table III, show that the original Thomas et al. (1985) approximation is very similar to our fit with CHIANTI and photospheric abundances for GOES 1-3, particularly for the temperature dependence, but that the parameters differ considerably for GOES 4 and later for the reasons discussed above.

To demonstrate the impact of the differences in the responses, Figure 8 compares the temperature and emission measure for a flare on 1998 June 11 derived using the CHIANTI/coronal response (solid lines), CHIANTI/photospheric response (dashed lines), the Mewe/Meyer response (dot-dash lines; obtained from the goes program in SolarSoft), and the Thomas et al. (1985) polynomial approximations. At the temperature peak of the flare during the rise phase of the X-ray flux, the two CHIANTI curves produce the same temperature: this is because the corresponding curves in the top panel of Fig. 7 cross at 15 MK. The other two responses give consistently lower temperatures, by about 1 MK for the Mewe/Meyer response and $2 \mathrm{MK}$ for the Thomas et al. (1985) approximations. At lower temperatures the CHIANTI/photospheric values are also about $1 \mathrm{MK}$ below the coronal abundance temperatures.

The higher temperatures found with coronal abundances in this event compound the fact that the coronal abundance response in the lower energy channel $b_{8}$ is larger than the corresponding photospheric abundance response and the fact that the higher temperature makes $b_{8}$ larger as well (Fig. 7, lower panel). This compound effect leads to emission measures that differ by more than the difference between the coronal and photospheric abundance curves in the lower panel of Fig. 7 would suggest, as shown in the lower panel of Fig. 8 . The Mewe/Meyer and Thomas et al. (1985) responses give very similar results, but the CHIANTI/photospheric emission measure is about $40 \%$ lower and the CHIANTI/coronal emission measure is about half of the photospheric emission measure. Thus in this event the assumption of coronal abundances together with the use of modern spectral models leads to emission measures that are a factor of order 4 smaller than current models predict in the case of $<15 \mathrm{MK}$ flares. At the peak of the flare the total thermal energy of the soft $\mathrm{X}$-ray plasma implied by the Mewe/Meyer response is 3 times the total energy derived from the CHIANTI coronal-abundance response, and about 50\% larger than for the CHIANTI photospheric-abundance response.

Note, however, that at temperatures above 15 MK the opposite effect occurs: for a given ratio of the fluxes in the two channels a smaller temperature will be deduced for coronal abundance than for photospheric abundance, and this will lead to a smaller difference in 


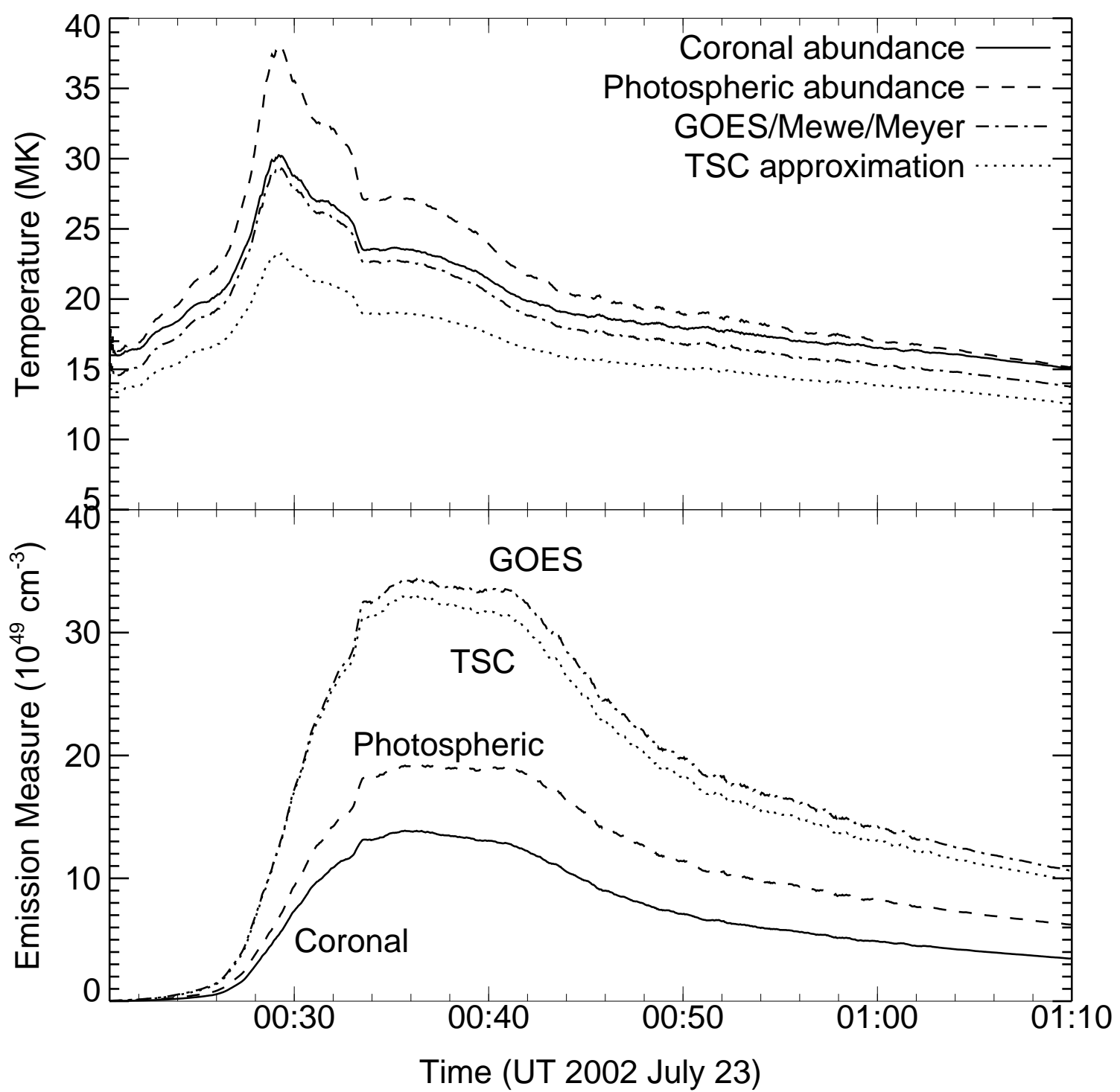

Figure 9. Comparison of the temperature (upper panel) and emission measure (lower panel) derived from GOES soft X-ray data for a flare on 2002 July 23, in the same format as Fig. 8. The data (and the responses used) are from the GOES 8 detectors.

the derived emission measure. We illustrate this in Figure 9 with the well-known flare of 2002 July 23, which produced much hotter soft X-rays than the previous example. The peak temperature in this event reached about $30 \mathrm{MK}$, and at these hotter temperatures the photospheric abundance response yields higher temperature determinations than does the coronal abundance response. The resulting derived emission measures show a difference of less than a factor of 2 between the coronal and photospheric abundance CHIANTI models, but again both are much smaller than the older responses would suggest. 


\section{Implementation}

The GOES XRS responses calculated using CHIANTI models and both coronal and photospheric abundances for each satellite will be made available through the SolarSoft goes package via a routine goes_chianti_tem that converts GOES XRS fluxes in the two channels into a temperature and emission measure under the isothermal assumption. The goes widget widely used in SolarSoft to retrieve GOES data and to carry out the conversion to temperature and emission measure will employ this routine and have options added to allow the selection of photospheric or coronal abundances in carrying out the conversion. The "hybrid abundance" (Fludra and Schmelz, 1999) case will lie somewhere between the photospheric and coronal values. The actual coefficients used to do the conversion are contained in routines called goes_get_chianti_temp and goes_get_chianti_em, while the GOES XRS transfer functions can be found in the routine goes_transfer.pro.

\section{Conclusions}

We have investigated the temperature response of the soft $\mathrm{X}$-ray flux measurements from NOAA's GOES series of satellites using modern CHIANTI spectral models with Mazzotta et al. (1998) ionization equilibria. We have used model spectra for a range of appropriate temperatures with both coronal and photospheric abundances to determine the response of the GOES XRS detectors as a function of temperature, and these responses may be used to determine temperatures and emission measures under the isothermal assumption. Although the detectors on the individual GOES satellites are quite similar, their data are calibrated in a satellite-dependent fashion and consequently the responses differ from one satellite to another.

An important and perhaps surprising result of this study is that the derived temperature and emission measure depend strongly on the assumed abundances even at high temperatures where continuum rather than spectral lines dominate the Sun's X-ray spectrum. For the currently accepted set of coronal abundances, most of the continuum is due to free-bound emission processes, not free-free emission, and thus is abundance-dependent. We find significant differences between modern calculations of the temperature response of the detectors and the versions currently in use: for a typical flare, emission measures can be up to a factor of 4 smaller than the current software suggests. Derived temperatures are similar for both photospheric and coronal abundances for cool flares (e.g., $15 \mathrm{MK}$ ), but for hot flares (e.g., 35 MK) coronal abundances can lead to significantly $(\sim 25 \%)$ lower temperatures being derived.

\section{Acknowledgements}

At the University of Maryland this research was supported by NSF grant ATM 02-33907 and NASA grants NAG 5-11872, NAG 5-12860 and NAG 5-10175. We thank Dr. Rodney Viereck of NOAA for supplying the calibration data for the GOES 12 detectors, Brian Dennis, Ken Phillips and Enrico Landi for discussions and encouragement, Hugh Hudson and Jim McTiernan for advice, Enrico Landi for supplying a model CHIANTI 5.0 spectrum in advance of release for comparison, and the referee Howard Garcia for valuable comments on the manuscript. 


\section{Appendix}

As described by Thomas et al. (1985) and Garcia (1994), the GOES soft X-ray detectors respond to the incident $\mathrm{X}$-ray flux by producing a current. The measured currents are converted to the reported $\mathrm{X}$-ray flux values by dividing the currents by the parameters $\overline{\mathrm{G}}_{4}$ $(0.5-4 \AA)$ and $\overline{\mathrm{G}}_{8}(1-8 \AA)$ that nominally represent wavelength-averaged transfer functions, i.e., averages over the transfer functions shown in Figure 1. (In practice the current data are actually telemetered to the ground as voltages that are proportional to the currents and the voltages are converted to fluxes in a single operation: see Garcia, 1994). The following table shows the values currently in use, as well as the nominal operational period for each spacecraft and the period for which we can find data available at the National Geophysical Data Center and/or the Solar Data Analysis Center. The value for $\overline{\mathrm{G}}_{8}$ shown for GOES 6 is the value that applies after June 28, 1993; prior to that date the value $4.43 \times 10^{-6}$ was used for the conversion of current to flux. Note that for their calculations for GOES 1 Thomas et al. (1985) used values slightly smaller than shown in the table for both $\overline{\mathrm{G}}_{4}$ and $\overline{\mathrm{G}}_{8}$; their values of $1.16 \times 10^{-5}$ and $3.5 \times 10^{-6}$, respectively, differ from the official values by some $10-15 \%$.

Table I. $\overline{\mathrm{G}}$ normalization coefficients for converting detector currents to X-ray fluxes, as well as the nominal operational period for each spacecraft and the period for which we can find data available at the National Geophysical Data Center and/or the Solar Data Analysis Center.

\begin{tabular}{lllll}
\hline Satellite & Operational & XRS data available & $\overline{\mathrm{G}}_{4}\left(\mathrm{~A} / \mathrm{W} \mathrm{\textrm {m } ^ { - 2 }}\right)$ & $\overline{\mathrm{G}}_{8}\left(\mathrm{~A} / \mathrm{W} \mathrm{m}^{-2}\right)$ \\
\hline GOES 1 & $1975 / 10 / 16-1985 / 03 / 07$ & $1976 / 01-1978 / 05$ & $1.27 \times 10^{-5}$ & $4.09 \times 10^{-6}$ \\
GOES 2 & $1977 / 06 / 16-1993$ & $1977 / 08-1983 / 05$ & $1.25 \times 10^{-5}$ & $3.98 \times 10^{-6}$ \\
GOES 3 & $1978 / 06 / 16-1993$ & $1978 / 07-1980 / 08$ & $1.25 \times 10^{-5}$ & $3.98 \times 10^{-6}$ \\
GOES 4 & $1980 / 09 / 09-1988 / 11 / 22$ & & $1.73 \times 10^{-5}$ & $4.56 \times 10^{-6}$ \\
GOES 5 & $1981 / 05 / 22-1990 / 07 / 18$ & $1983 / 01-1987 / 02$ & $1.74 \times 10^{-5}$ & $4.84 \times 10^{-6}$ \\
GOES 6 & $1983 / 04 / 28-$ & $1983 / 05-1994 / 08 / 18$ & $1.74 \times 10^{-5}$ & $5.32 \times 10^{-6}$ \\
GOES 7 & $1987 / 02 / 26-1996 / 08 / 03$ & $1987 / 03-1996 / 08 / 03$ & $1.68 \times 10^{-5}$ & $4.48 \times 10^{-6}$ \\
GOES 8 & $1994 / 04 / 13-2004 / 05 / 05$ & $1996 / 03 / 21-2003 / 06 / 18$ & $1.580 \times 10^{-5}$ & $4.165 \times 10^{-6}$ \\
GOES 9 & $1995 / 05 / 23-$ & $1996 / 03 / 20-1998 / 09 / 08$ & $1.607 \times 10^{-5}$ & $3.990 \times 10^{-6}$ \\
GOES 10 & $1997 / 04 / 25-$ & $1998 / 07 / 10-$ & $1.631 \times 10^{-5}$ & $3.824 \times 10^{-6}$ \\
GOES 11 & $2000 / 05 / 03-$ & Backup & & $1.595 \times 10^{-5}$ \\
GOES 12 & $2001 / 07 / 23-$ & $2002 / 12 / 13-$ & $4.090 \times 10^{-6}$ \\
\hline
\end{tabular}

Thomas et al. (1985) derived polynomial approximations that allow one to derive the temperature, $T$, and volume emission measure, $E M=N_{e} N_{H} V$, from the ratio $R=B_{4} / B_{8}$ of the reported X-ray fluxes $B_{4}(0.5-4 \AA)$ and $B_{8}(1-8 \AA)$, and the value of $B_{8}$ as follows: the temperature is obtained from

$$
T(R)=A(0)+A(1) R+A(2) R^{2}+A(3) R^{3}
$$

and once the temperature is known the emission measure is derived from $E M=10^{55} B_{8} / b_{8}(T)$ $\mathrm{cm}^{-3}$ where $B_{8}$ is the 1-8 $\AA$ flux in the standard GOES unit of $\mathrm{W} \mathrm{m}^{-2}$ and

$$
b_{8}(T)=B(0)+B(1) T+B(2) T^{2}+B(3) T^{3}
$$


is the normalized response.

We have derived such polynomial approximations for all GOES satellites using CHIANTI spectral models as discussed above. The next two tables present the $A(0-3)$ and $B(0-3)$ coefficients for each satellite, as well as the original Thomas et al. (1985) values (labelled "TSC"). The next table presents the coefficients under the assumption of coronal abundances, while the last table presents the coefficients for photospheric abundances. They result from fitting curves such as those shown in Figure 7 ( $T$ versus $R$ and $b_{8}$ versus $T$ for GOES 10) over the temperature range 4-40 MK. The fits work well in the range 5-40 MK: temperatures are always correct to within $4 \%$ (coronal abundances) or $2 \%$ (photospheric abundances) and emission measures are correct to within $9 \%$ (coronal) or $8 \%$ (photospheric) across this range, with the errors largest at low temperatures where the $B_{4} / B_{8}$ ratio is very small. In the range 10-40 MK which is appropriate for most solar flares, the errors in the emission measure are always less than $4 \%$. However, the tabulated data used in the IDL routines available through SolarSoft are more accurate than these polynomial approximations and should be used whenever possible.

Table II. Coefficients for polynomial approximations to GOES temperature response: Coronal abundances

\begin{tabular}{l|rrrr|rrrr}
\hline Satellite & $\mathrm{A}(0)$ & $\mathrm{A}(1)$ & $\mathrm{A}(2)$ & $\mathrm{A}(3)$ & $\mathrm{B}(0)$ & $\mathrm{B}(1)$ & $\mathrm{B}(2)$ & $\mathrm{B}(3)$ \\
\hline TSC & 3.15 & 77.2 & -164.0 & 205.0 & -3.86 & 1.17 & -0.0131 & 0.000178 \\
GOES 1 & 3.74 & 77.5 & -158.0 & 180.3 & -12.80 & 3.88 & -0.1020 & 0.001210 \\
GOES 2 & 3.74 & 78.3 & -161.7 & 186.6 & -13.15 & 3.99 & -0.1048 & 0.001243 \\
GOES 3 & 3.74 & 78.3 & -161.7 & 186.6 & -13.15 & 3.99 & -0.1048 & 0.001243 \\
GOES 4 & 3.74 & 94.6 & -235.9 & 328.9 & -11.48 & 3.48 & -0.0915 & 0.001085 \\
GOES 5 & 3.74 & 89.7 & -211.8 & 279.8 & -10.81 & 3.28 & -0.0862 & 0.001022 \\
GOES 6 & 3.83 & 86.2 & -193.3 & 242.1 & -10.25 & 3.12 & -0.0836 & 0.000997 \\
GOES 7 & 3.68 & 101.2 & -271.3 & 409.3 & -11.87 & 3.54 & -0.0882 & 0.001027 \\
GOES 8 & 4.02 & 100.3 & -258.1 & 366.5 & -12.56 & 3.87 & -0.1077 & 0.001298 \\
GOES 9 & 3.97 & 100.8 & -260.7 & 373.1 & -12.63 & 3.91 & -0.1097 & 0.001326 \\
GOES 10 & 3.81 & 101.5 & -270.7 & 404.6 & -12.03 & 3.61 & -0.0928 & 0.001091 \\
GOES 12 & 3.90 & 101.2 & -266.4 & 390.2 & -12.31 & 3.75 & -0.1003 & 0.001195 \\
\hline
\end{tabular}


Table III. Coefficients for polynomial approximations to GOES temperature response: Photospheric abundances

\begin{tabular}{l|llll|lllr}
\hline Satellite & $\mathrm{A}(0)$ & $\mathrm{A}(1)$ & $\mathrm{A}(2)$ & $\mathrm{A}(3)$ & $\mathrm{B}(0)$ & $\mathrm{B}(1)$ & $\mathrm{B}(2)$ & $\mathrm{B}(3)$ \\
\hline TSC & 3.15 & 77.2 & -164.0 & 205.0 & -3.86 & 1.17 & -0.0131 & 0.000178 \\
GOES 1 & 3.06 & 75.3 & -145.9 & 210.9 & -5.70 & 1.59 & -0.0169 & 0.000110 \\
GOES 2 & 3.06 & 76.2 & -149.3 & 218.3 & -5.85 & 1.63 & -0.0174 & 0.000113 \\
GOES 3 & 3.06 & 76.2 & -149.3 & 218.3 & -5.85 & 1.63 & -0.0174 & 0.000113 \\
GOES 4 & 3.06 & 92.0 & -217.9 & 384.7 & -5.11 & 1.42 & -0.0152 & 0.000099 \\
GOES 5 & 3.06 & 87.2 & -195.6 & 327.4 & -4.81 & 1.34 & -0.0143 & 0.000093 \\
GOES 6 & 3.15 & 84.1 & -178.9 & 283.3 & -4.56 & 1.28 & -0.0145 & 0.000101 \\
GOES 7 & 2.98 & 98.8 & -250.6 & 478.2 & -5.33 & 1.45 & -0.0129 & 0.000062 \\
GOES 8 & 3.36 & 98.6 & -242.0 & 434.0 & -5.54 & 1.58 & -0.0201 & 0.000158 \\
GOES 9 & 3.31 & 98.6 & -242.7 & 439.2 & -5.56 & 1.59 & -0.0208 & 0.000168 \\
GOES 10 & 3.15 & 99.2 & -250.9 & 473.8 & -5.37 & 1.48 & -0.0145 & 0.000084 \\
GOES 12 & 3.24 & 99.2 & -247.9 & 458.8 & -5.47 & 1.53 & -0.0173 & 0.000121 \\
\hline
\end{tabular}




\section{References}

Aschwanden, M. J. and D. Alexander: 2001. Solar Phys. 204, 91-120.

Bornmann, P. L.: 1990. Astrophys. J. 356, 733-742.

Brown, G. V., P. Beiersdorfer, D. A. Liedahl, K. Widmann, S. M. Kahn, and E. J. Clothiaux: 2002. Astrophys. J. Supp. 140, 589-607.

Dere, K. P., E. Landi, , H. E. Mason, B. C. Monsignori Fossi, and P. R. Young: 1997. Astron. Astrophys. Supp. Ser. 125, 149.

Feldman, U.: 1992. Physica Scripta 46, 202.

Feldman, U. and J. M. Laming: 2000. Physica Scripta 61, 222.

Feldman, U. and K. G. Widing: 2003. Space Science Reviews 107, 665-720.

Fludra, A. and J. T. Schmelz: 1999. Astron. Astrophys. 348, 286.

Freeland, S. L. and B. N. Handy: 1998. Solar Phys. 182, 497-500.

Garcia, H. A.: 1994. Solar Phys. 154, 275-308.

Garcia, H. A.: 2004. Space Weather 2, S06003.

Gu, M. F., S. M. Kahn, D. W. Savin, E. Behar, P. Beiersdorfer, G. V. Brown, D. A. Liedahl, and K. J. Reed: 2001. Astrophys. J. 563, 462-471.

Jordan, C.: 1970. Mon. Not. Roy. Astron. Soc. 148, 17.

Landi, E., U. Feldman, and K. P. Dere: 2002. Astrophys. J. Supp. 139, 281-296.

Landi, E., M. Landini, K. P. Dere, P. R. Young, and H. E. Mason: 1999. Astron. Astrophys. Supp. Ser. 135, 339.

Liedahl, D. A., A. L. Osterheld, and W. H. Goldstein: 1995. Astrophys. J. (Lett.) 438, L115-L118.

Mazzotta, P., G. Mazzitelli, S. Colafrancesco, and N. Vittorio: 1998. Astron. Astrophys. Supp. Ser. 133, 403-409.

Mewe, R., E. H. B. M. Gronenschild, and G. H. J. van den Oord: 1985. Astrophys. J. Supp. 62, 197.

Meyer, J.-P.: 1985. Astrophys. J. Supp. 57, 173.

Phillips, K. J. H.: 2004. Astrophys. J. 605, 921-930.

Phillips, K. J. H. and U. Feldman: 1995. Astron. Astrophys. 304, 563.

Raymond, J. C. and B. W. Smith: 1977. ApJS 35, 419.

Savin, D. W., P. Beiersdorfer, J. C. Lopez-Urrutia, V. Decaux, E. M. Gullikson, S. M. Kahn, D. A. Liedahl, K. J. Reed, and K. Widmann: 1996. Astrophys. J. (Lett.) 470, L73.

Schmelz, J. T.: 1999. In: ESA SP-446: 8th SOHO Workshop: Plasma Dynamics and Diagnostics in the Solar Transition Region and Corona. p. 585.

Sylwester, J., H. A. Garcia, and B. Sylwester: 1995. Astron. Astrophys. 293, 577-585.

Thomas, R. J., R. Starr, and C.-J. Crannell: 1985. Solar Phys. 95, 323.

Veronig, A. M., J. C. Brown, B. R. Dennis, R. A. Schwartz, L. Sui, and A. K. Tolbert: 2005. Astrophys. J. in press.

Walker, Jr., A. B. C., H. R. Rugge, and K. Weiss: 1974. Astrophys. J. 188, 423.

White, S. M., R. Thomas, J. W. Brosius, and M. R. Kundu: 2000. Astrophys. J. (Lett.) 534, L203.

Young, P. R., G. del Zanna, E. Landi, , K. P. Dere, H. E. Mason, and M. Landini: 2003. Astrophys. J. Supp. $144,135$.

Young, P. R., E. Landi, and R. J. Thomas: 1998. Astron. Astrophys. 329, 291. 